\title{
A Corpus-Based Study of the Phraseological Pattern It + V-link + ADJ + That Clause in L1 and L2 Expert Academic Writing
}

Richmond Sadick Ngula*

Department of English, University of Cape Coast, Cape Coast, Ghana

Corresponding Author: Richmond Sadick Ngula, E-mail: rngula@ucc.edu.gh

\begin{tabular}{l} 
ARTICLE INFO \\
\hline Article history \\
Received: March 04, 2018 \\
Accepted: May 05, 2018 \\
Published: September 01, 2018 \\
Volume: 7 Issue: 5 \\
Advance access: July 2018
\end{tabular}

Conflicts of interest: None Funding: None

\begin{abstract}
This study discusses the phraseological pattern $I t+V$-link $+A D J+$ That Clause as a rhetorical feature of argumentation in Ghanaian scholars' research articles (RAs) across the disciplines of Sociology, Economics and Law. It looks at the pattern's occurrence with the adjectives possible, likely and clear in RAs by Ghanaian authors based in Ghana and in RAs by international scholars who are native speakers to determine potential divergent patterns of use between the two groups of scholars in the three disciplinary fields. Because RAs produced by non-native writers are often said to be characterized by overuse, underuse or misuse of rhetorical features (e.g., Martinéz, 2005, Englander, 2006), this study adopts a corpus-based approach to investigate the extent to which Ghanaian scholars' use of the above collocational pattern involving possible, likely and clear differs from international scholars' use of the pattern in terms of frequency information, levels of epistemic force, and intensification/mitigation styles. A close inspection of a millionword corpus of RAs, supported by robust statistical analyses, reveals considerable differences in the way the pattern is used between the two groups of scholars across the disciplines studied, which suggest that Ghanaian scholars do not fully apply the preferred stereotypical uses of the pattern found in reputable international RAs. The study has implications for how Ghanaian scholars have acquired rhetorical strategies of academic writing.
\end{abstract}

Key words: Phraseology and Patterns, Academic Writing, Epistemic Modality, Research Articles, Rhetoric

\section{INTRODUCTION}

Phraseological patterns of lexical devices have been shown to be of considerable importance in academic discourse, especially in written academic texts. The role of such patterns in marking interpersonal and dialogic links - to enhance our understanding of how writers and readers negotiate the dialogic space - has recently attracted intense scholarly interest, although the field of phraseology itself [for a long time] "has been a neglected area of language description" (Sinclair, 2008: xv).

Studies of phraseological units within academic discourse include Charles (2006) who looked at patterns in reporting clauses in theses, Laso and John (2013) who explored co-occurrence between adjectives and abstract nouns in medical English; Ventura (2013) who studied the phraseology of the verb 'describe' in health science research articles; and Peacock (2012) who explored noun collocations in research articles in eight disciplines. Such studies have largely been inspired by Sinclair's $(1987,1991,2004)$ conceptualization and development of collocation theory, stressing what he calls the 'idiom principle'. According to Sinclair (1987: 320), "[t]he principle of idiom is that a language user has available to him or her a large number of semi-preconstructed phrases that constitute single choices, even though they might appear to be analyzable into segments". This is to suggest the intricate relationship between single words and their syntactic co-occurrence, and the role such a relationship plays in the interpretation of meaning in texts. Thus, meaning in language is based on recurrent phraseological units rather than on single words on their own (Hunston and Francis, 2000; Römer, 2009).

Studies of co-occurrence patterns in academic discourse, such as those mentioned above, not only shed light on the stereotypical phraseological constructions associated with specific academic communities, they also offer valuable information for academic writing pedagogy, and writers who use English as an additional language to familiarize themselves with disciplinary community practices. For research articles (RAs) specifically, Laso, Comelles and Verdaguer (2013: 105) note that "formulaic constructions ... need to be mastered by the members of the scientific community, so as to learn the preferred ways of conveying information and thus produce phraseologically competent research articles". Implied in this view by Laso et al. is that there are dispreferred and inappropriate ways of academic communication 
which may be frowned upon by the more experienced members of disciplinary communities.

In this article, I explore the phraseological pattern $I t+$ V-link + ADJ + That Clause (with the adjectives possible, likely, and clear in the pattern) in research articles (RAs) written in English by two different groups of scholars: Ghanaian multilingual scholars in the disciplines of Sociology, Economics and Law (L2), and international scholars in similar disciplines who are native speakers (L1). My interest in this study lies with the Ghanaian scholars who are based in Ghana and writing and publishing quite considerably in locally-based English-medium journals, yet their overall contribution to mainstream international disciplinary communities is very limited. Thus, we might consider the international scholars represented in this study as the control group whose expert writing, in the form of RAs, captures what may be regarded as the preferred stereotypical practices and conventions for international communities of the three disciplines. I will therefore be concerned with the extent to which the Ghanaian L2 scholars' use of the pattern It $+V$-link + ADJ + That Clause compares with its use by the international L1 scholars in terms of frequency of use, intensification/mitigation styles, and epistemic force.

A current area of scholarly interest within English for Academic Purposes (EAP) has to do with the writing challenges and difficulties of multilingual scholars who are non-native speakers of English and who operate from less-advantaged geographical contexts. These contexts are often characterized in the academic world as "off network" and "periphery" and contrasted with the more mainstream and center-based contexts of scholarship, i.e. the Anglo-American contexts (cf. Canagarajah, 1996, 2002; Swales, 2004; Salager-Meyer, 2008). Much of the research targeting non-native multilingual scholars' use of rhetorical features of argumentation in academic discourse (Flowerdew, 1999; Martinéz, 2005; Englander, 2006; Leki et al., 2008) reports on inappropriate linguistic and rhetorical choices such as overuse, underuse and misuse. Martinéz (2005: 176), for example, claims that multilingual scholars who write articles in English need to be empowered to become more "aware of the variety of linguistic resources upon which NES writers draw". And according to Leki et al. (2008: 58), L2 scholars writing in English are confronted with several obstacles, including the "need to control linguistic and rhetorical features of English".

Remarks such as those by Martinéz and Leki et al. are the outcome of the analysis of textual and survey data produced by non-native English writers. Much of the work, however, has concentrated on non-English speaking writers in Europe, Asia and Latin America. There is very little work on the topic in Africa, with Nkemleke's (2010) article on Cameroon scholars being a notable exception). In Ghana specifically, where English is an important L2 with official status, scholars are writing and publishing considerably in English, but their writing practices are yet to be empirically studied. Academic writing research in Ghana has concentrated on students' writing (e.g., Dako, 1997; Adika, 1999; Anyidoho, 2002; Afful, 2005), resulting in a dearth of research on scholarly writing. I would like to express the view that in
English as a Second Language (ESL) contexts such as Ghana, studies on the linguistic and rhetorical features of professional scholarly writing should begin to receive the needed attention as these may have direct practical and pedagogical benefits for Ghanaian practitioners and novice writers (including students) alike.

In this article, I focus on the rhetorical practices of Ghanaian scholars in the disciplines of Sociology, Economics and Law. Does their use of the pattern under study $-I t+V$-link $+A D J+$ That Clause - offer additional evidence of overuse, underuse or misuse of rhetorical markers of argumentation? And might their 'off-network' and 'periphery' status suggest that they are less aware of centre-based norms of scholarly writing? In the remainder of the article, the pattern $I t+$ $V$-link $+A D J+$ That Clause and its role as an epistemic resource in academic writing are explored, the corpus data and methods used for the study are described, the analysis and findings of the use of the pattern are discussed, focusing on how frequency information, intensification/mitigation styles and levels of epistemic force might vary across the disciplines and between the Ghanaian and international scholars. In the conclusion the theoretical and pedagogical implications of the study are discussed.

\section{$I T+V-L I N K+A D J+T H A T C L A U S E$ AS AN EPISTEMIC MODALITY RESOURCE}

Epistemic modality relates to the deductive conclusions or claims we make when we write or speak, basing them on the state of our evidence or knowledge of situations or circumstances. It is this evidence - available to a writer or speaker - that regulates the level of confidence or force backing a claim, statement or conclusion. According to Vold (2006: 226), "epistemic modality concerns the reliability of the information conveyed, and epistemic modality markers can be defined as linguistic expressions that explicitly qualify the truth value of a propositional content". English has a wide range of resources to mark epistemic modality, including modal verbs (e.g., will, can, may, must, could), which are perhaps the most common resource, lexical verbs (e.g., seem, suggest), adjectives (e.g., possible, obvious), nouns (e.g., chance, probability), adverbs (e.g., perhaps, possibly), and non-lexical resources like phrases and other syntactic combinations (cf. Biber et al., 1999).

In this paper I focus on epistemic adjectives in the phraseology It $+V$-link + ADJ + That Clause. This pattern conveys a variety of epistemic meanings, especially when certain adjectives (such as the ones explored in the present study - possible, (un)likely, clear) are used. While it does not contain a modal verb, which is the primary resource for expressing (epistemic) modality, the pattern is one of those structures which carry an epistemic meaning. Hunston (2011) has termed these structures 'modal-like expressions', using examples like It is obvious/apparent/evident/doubtful/ possible that ... to illustrate the It + V-link + ADJ + That Clause pattern. But she explains also that the pattern may have in it other evaluative, non-epistemic adjectives such as It is relevant/essential/acceptable/awful/critical that ... etc. (see Hunston, 2011: 139). This study does not consider 
non-epistemic adjectives in this pattern. Indeed, as I point out above, my focus in this pattern is on the three epistemic adjectives possible, (un)likely and clear. Two reasons explain why I focus on these three: the first is that they are the most frequently used epistemic items of this pattern in the corpus of RAs I studied, and the second is that they fit in well in terms of the three-way scale of epistemic force/commitment adopted in this study; i.e. weak (possible), medium ((un)likely) and strong (clear), a scale type that has been widely applied (e.g., Hyland and Milton, 1997; Nuyts, 2001; McEnery and Kifle, 2002; Biber, Conrad \& Leech, 2002). The following section describes the corpus data used for this study and the procedures of analysis followed to arrive at the findings reported in this paper.

\section{CORPUS DATA AND METHODOLOGY}

\section{The Corpus}

The computer specialised corpus used for this study was made up of a collection of two sets of RA samples, one for the international scholars in the three disciplines of Sociology, Economics and Law, and the other for the Ghanaian scholars in the same disciplines. The international scholars' sub corpus contained 20 RAs for Sociology (147, 912 tokens), 20 RAs for Economics (148, 926 tokens), and 20 RAs for Law (221, 608 tokens). The Ghanaian scholars' sub corpus contained 23 RAs for Sociology (132, 676 tokens), 23 RAs for Economics (145, 892 tokens), and 23 RAs for Law (168, 922 tokens). I collected more RA samples (23 articles against 20 for the international corpus) for the Ghana corpus because the Ghanaian scholars generally wrote shorter articles, and, even with these numbers, the tokens of the RAs by the international scholars, as mentioned above, were slightly more than those for the Ghanaian scholars. To ensure that this disparity in tokens was negligible, the frequency results presented in this study were normalized to a base of 10,000 tokens. Overall, I worked with a corpus of approximately 1 million words. The RAs collected and processed for the corpus were all published from 2000 to 2010/11 and appeared in the journal sources listed in Table 1 which summarises the main features of the sub corpora.

Overall, the corpora representing the two groups of scholars were very similar and therefore comparable, although practical decisions carried out during text collection and processing led to slight changes in the original corpus design. It is unlikely that these minor changes affected the analysis of the epistemic forms and patterns explored in this study. The practical challenges experienced on the field during the construction of the sub corpora of RAs reflect McEnery, Xiao and Tono's (2006: 73) view that "corpus building is of necessity a marriage of perfection and pragmatism". (For further details about the sub corpora used for this study see Ngula, 2015.)

\section{Analysis}

The starting point for the analysis was to get a sense of the frequency of occurrence of the epistemic adjectives - possible, likely and clear - between the two groups of scholars in the three disciplines. As these three resources are key examples of epistemic devices at the weak, medium and strong levels respectively, their frequency of occurrence in the RAs studied immediately gives an indication of the extent to which epistemic devices, both overall and at each of these three levels, are exploited by the two groups of writers. Frequencies are based not only on normed figures (percentage scores), but also on whether raw frequency differences of use, either across disciplinary fields or between the Ghana and international writers, are statistically significant or not. I used Dunning's (1993) log-likelihood test, specifically the calculator version designed by Paul Rayson and available online at http://ucrel.lancs.au.uk/llwizard.html, to compute the figures. To be sure that I could report confidently any observed difference in the use of a linguistic feature, as being

Table 1. Corpora used in the study

\begin{tabular}{|c|c|c|}
\hline Discipline & Ghana (NNGC) & International (NAAC) \\
\hline Sociology & $\begin{array}{l}23 \text { articles } \\
132676 \text { tokens } \\
\text { Published: } 2000-2011 \\
\text { Source: e.g., legon journal of sociology, ghana social } \\
\text { science journal, oguaa journal of social sciences }\end{array}$ & $\begin{array}{l}20 \text { articles } \\
147912 \text { tokens } \\
\text { Published: } 2000-2010 \\
\text { Source: e.g., American journal of sociology, the british } \\
\text { journal ofsSociology, the sociological review, gender and } \\
\text { society }\end{array}$ \\
\hline Economics & $\begin{array}{l}23 \text { articles } \\
145892 \text { tokens } \\
\text { Published: } 2000-2011 \\
\text { Source: e.g., journal of economics studies, ghana } \\
\text { policy journal, ghana social science journal, oguaa } \\
\text { journal of social sciences }\end{array}$ & $\begin{array}{l}20 \text { articles } \\
148926 \text { tokens } \\
\text { Published: } 2000-2010 \\
\text { Source: e.g., The quarterly journal of economics, the review } \\
\text { of economic studies, journal of economic perspectives, } \\
\text { labour economics }\end{array}$ \\
\hline Law & $\begin{array}{l}23 \text { articles } \\
168922 \text { tokens } \\
\text { Published: } 2000-2011 \\
\text { Source: University of ghana law journal, the Review } \\
\text { of ghana Law, The KNUST law journal }\end{array}$ & $\begin{array}{l}20 \text { articles } \\
221608 \text { tokens } \\
\text { Published: } 2000-2010 \\
\text { Source: e.g., The American journal of international law, } \\
\text { the European journal of international law, oxford journal of } \\
\text { legal studies, international journal of constitutional law }\end{array}$ \\
\hline
\end{tabular}


statistically significant or not, I set the log-likelihood significance level to $\mathrm{p}<0.01$ (with a critical value of 6.63 ).

Following the overall frequency analysis, each of the three adjective resources is examined qualitatively for their patterns of phraseology via a scrutiny and close examination of concordance lines. Every concordance output is sorted in various ways to enable patterns to become more obvious. Adjectives have been shown to be an important group of lexical resources that produce considerable phraseological patterns (Hunston and Francis, 1999; Hunston, 2002; Groom, 2005), and in this study, they contribute in shaping the epistemic meanings adjectives encode in RAs.

\section{RESULTS AND DISCUSSION}

\section{Overall frequency of possible, likely, clear}

I begin by looking at what can be reported with regards to the general frequency patterns of possible, likely and clear as epistemic adjectives in the RAs studied. Table 1 summarises the overall occurrences of these epistemic devices in the corpus of RAs of the two groups of scholars for the three disciplines. While the raw frequencies are presented alongside the normalised ones in brackets (occurrences per 10, 000 running words), the discussion of the results is based on the normed frequencies. The raw figures have helped to calculate the Log-likelihood values used to determine whether the frequency differences recorded for the two groups of scholars are statistically significant or not.

If we consider the overall figures, as can be seen in Table 2, likely, a medium-level epistemic resource, has a high occurrence in the RAs written by both groups of scholars, although the international scholars (IS) in all three disciplines use it significantly more than the Ghanaian scholars (GS). Between possible and clear, a different frequency pattern emerges for the two groups of scholars: the IS across all three disciplines use possible (a weak epistemic device) slightly more than they do with clear (a strong epistemic device). However, the GS generally appear to prefer clear over possible as epistemic devices, despite that the Ghanaian Sociologists use possible a little more (0.83) than clear (0.53). It thus seems apparent that if we compare the overall uses of these three epistemic devices between the IS and the GS, while the IS are more inclined to move from a medium-level resource (likely) towards a weak-level resource (possible), and only sparingly using the strong-level epistemic adjective (clear), the GS seem to prefer a movement from a medium-level resource (likely) towards a strong-level resource (clear), and only minimally exploiting the weak-level epistemic resource (possible) to make and advance their arguments. This further suggests that the IS are generally more cautious in the way they report research claims, compared to the GS, who, from the overall figures in Table 1, can be said to underuse the epistemic adjective likely and possible. This can also be seen in the relatively high use of the strong form clear, which, while being equally used by both the IS and GS in Economics and Law, is used significantly more by the GS than by the IS.

The dramatic underuse of the epistemic forms likely and possibly by the GS confirms the findings of several studies on the non-native speaker's use of epistemic modality in RAs (e.g., Englander, 2006; Panacová, 2008) and other academic genres such as the doctoral thesis (e.g., Ağçam, 2014), a confirmation which suggests that the GS report research claims in a more direct, unmitigated and overly categorical way.

\section{THE IT + V-LINK +ADJ + THAT CLAUSE PATTERN}

In the RAs written by the IS, a close analysis of concordance lines reveals that the epistemic devices possible, likely and clear, compared with - say - true and obvious, are more typical of the phraseological pattern it v-link ADJ that. If we consider clear, for example, sentences (1) and (2) illustrate its occurrence in this pattern in Sociology and Economics, which is used by the writers to make readers accept the claim being made as obvious and apparent.

1) It is also clear that each instantiation of 'being ethical' was presented by interviewees within a larger account of a particular context ... [SOC NA05]

2) By the late $1980 \mathrm{~s}$, it was clear that this scheme was becoming fiscally unsustainable. [ECO NA01]

With regards to possible and likely, it is important to note that the epistemic notions of 'possibility' and 'probability' inherent in them respectively are not particularly dependent on the words possible and likely per se; rather,

Table 2. Frequencies of possible, likely and clear in the pattern under study

\begin{tabular}{lcccc}
\hline & $\begin{array}{c}\text { International } \\
\text { scholars (IS) }\end{array}$ & $\begin{array}{c}\text { Ghanaian } \\
\text { scholars (GS) }\end{array}$ & $\begin{array}{c}\text { Log-likelihood figures at critical } \\
\text { Value }=\mathbf{6 . 6 3}\end{array}$ & $\begin{array}{c}\text { Significance } \\
\text { level: } \mathbf{P}<\mathbf{0 . 0 1}\end{array}$ \\
\hline possible (SOC) & $43(2.91)$ & $11(0.83)$ & 16.95 & Sig. \\
possible (ECO) & $62(4.16)$ & $30(2.06)$ & 10.72 & Sig. \\
possible (LAW) & $67(3.02)$ & $11(0.65)$ & 30.90 & Sig. \\
likely (SOC) & $198(13.39)$ & $56(4.22)$ & 69.46 & Sig. \\
likely (ECO) & $85(5.71)$ & $37(2.54)$ & 18.43 & Sig. \\
likely (LAW) & $113(5.10)$ & $37(2.19)$ & 22.47 & Sig. \\
clear (SOC) & $30(2.03)$ & $7(0.53)$ & 13.01 & Sig. \\
clear (ECO) & $35(2.35)$ & $37(2.54)$ & 0.10 & Not Sig. \\
clear (LAW) & $59(2.66)$ & $37(2.19)$ & 0.18 & Not Sig. \\
\hline
\end{tabular}


as Groom (2005) points out, the validity of the epistemic meanings is restricted by the pattern of focus (it $v$-link $A D J$ that), such that certain syntagmatic changes in this pattern can dramatically affect the epistemic meaning. Considering possible specifically, the epistemic meaning would change to a 'root possibility' sense if we replace the that-clause following $A D J$ with a to/for phrase or clause (See Coates, 1981), as in (3), which expresses a root rather than an epistemic possibility (glossed as something like 'one can cite several factors ...', which is clearly non-epistemic).

3) In the case of graduates, it is possible to cite several factors which might encourage a flexible use of their labour that might reduce the rewards to having a degree. [SOC NA04]

Example (3) suggests that the that-clause in the it v-link $A D J$ that pattern, like other parts of the syntagm, is equally important in deriving the epistemic meaning expressed by possible. In the RAs by the international scholars (IS), the it $v$-link ADJ that pattern is used by writers in all the three disciplines as a mitigating epistemic resource to signal a less confident posturing in the claim being made. In the IS RAs, possible in this pattern is represented in 8 concordance lines in Sociology, 13 in Economics and 11 in Law. While these (raw) frequencies are not large enough to account for any meaningful disciplinary variations, we can argue overall, in a qualitatively way, that the IS writers in these social science fields find the use of possible in this phraseological pattern a useful rhetorical strategy for negotiating research claims. Figure 1 represents a snapshot of the 13 concordance lines for the pattern it v-link possible that in the IS Economics RAs, for example.

If we turn to the form likely, a constraint of a different kind can be seen when it occurs in the pattern it v-link ADJ that. The epistemic meaning of likely in this pattern is one of probability, a much stronger epistemic force than epistemic possibility. When the pattern has likely as its $A D J$, it is less rigid. This is because the epistemic meaning of probability conveyed is less affected by the that-clause, unlike with possible, so that in the absence of the that-clause which follows the $A D J$, the epistemic sense is still intact, as in (4) taken from Law.

4) Provided that the vehicle has legal personality, it is likely to enjoy standing before ICSID. [LAW NA02]

But certain kinds of structural change in the occurrence of likely in this pattern could change the epistemic value.
A case in point involves the negation of likely in the pattern, expressed either as it is not likely that or in the form of derivational affixation (it is unlikely that). In this negation pattern, it is apparent that there is absence of probability, yet the pattern still encodes an epistemic meaning, one of doubt or improbability, which is weak (in terms of epistemic force) and far less certain. It essentially assumes the function of a hedge, as in (5) in Economics and (6) in Law.

5) Until then, given the very large gap between the cost-effectiveness estimates for prevention and treatment, it is not likely that correcting measurement errors will reverse the ranking. [ECO NA14]

6) It is unlikely that this fagade of administrative objectivity will serve for long to deflect judicial review. [LAW NA11]

Only a few instances of this negative pattern are found in the IS RAs examined, but the few examples noted also show that the international writers seem to prefer the affix negation type of (6) over the clausal negation type of (5). In total, I found 3 examples of the negation pattern in the IS Sociology RAs, all of the affix type; 7 examples in the IS Law RAs, all of the affix type; and 3 examples in the IS Economics RAs, where 2 are of the affix type and 1 of the clausal type (i.e. example 5 above).

Even though the international writers use this negative pattern, the positive use of likely in the pattern it v-link ADJ that to mark the epistemic meaning of probability remains an important feature of their RAs, especially in the field of Sociology. My corpus reveals 15 concordance lines with this pattern in the IS Sociology RAs, although there are far less examples in Economics (2 concordance lines) and Law (3 concordance lines). Thus, while qualitatively the it v-link likely that pattern is one that is found in the RAs of all three disciplines, the writers of Sociology seem likely to use it more than their colleagues in Economics and Law. However, I think that this preference can only be regarded as a tendency: to make firmer quantitative claims about such phraseological patterns across these social science disciplines would require much larger corpora for these disciplines. Figure 2 shows the 15 concordance lines for the pattern it v-link likely that-clause in the IS Sociology RAs.

A point of note in Figure 2 can be seen in lines 1115 where the v-link item 'seem' or 'would seem' enters a sort of epistemic harmony with the ADJ likely, a harmony which serves to mitigate or reduce the probability sense in this pattern.

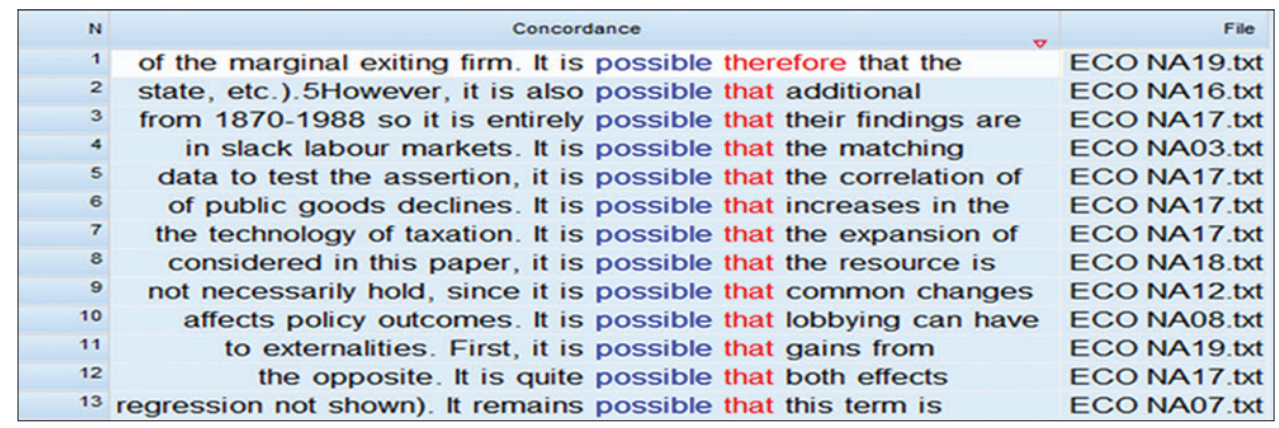

Figure 1. Concordance lines for the it $v$-link possible that pattern in the IS Economics RAs 


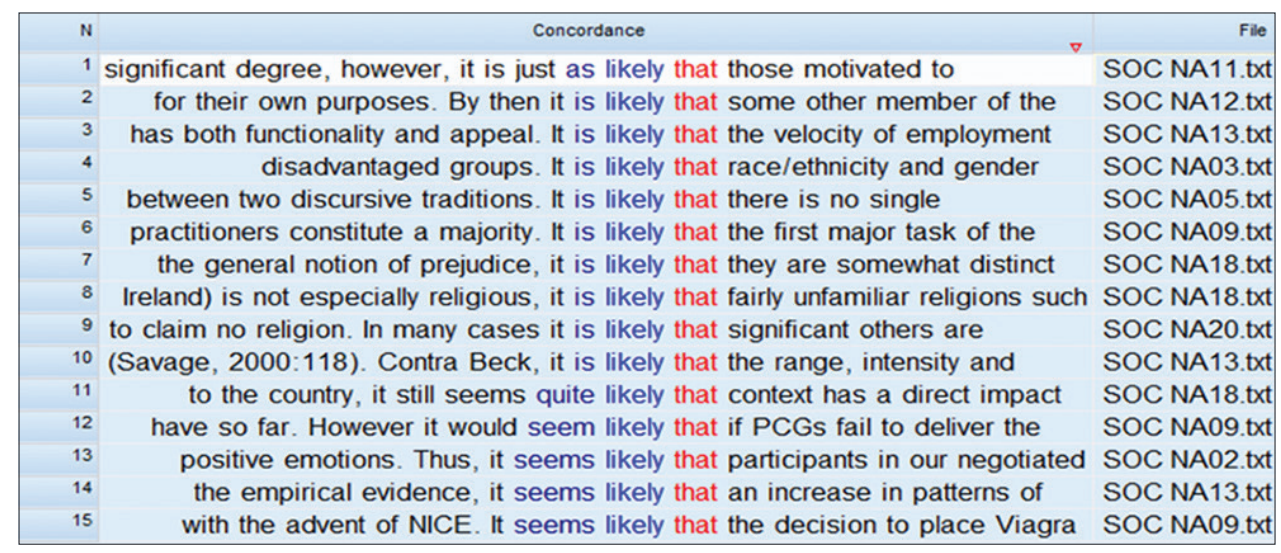

Figure 2. Concordance lines for the it v-link likely that pattern in the IS Sociology RAs

So far, I have been looking at the pattern of focus in the corpus of RAs representing the IS. I turn now to the Ghanaian scholars and their use of the it v-link ADJ that pattern. This phraseological pattern is encountered in the RAs written by the GS too, although some noticeable differences are seen in comparison with the RAs written by the IS. First, while in the IS RAs the adjectives possible, likely and clear are all quite common in the pattern, the GS RAs tend to have clear alone standing out as the most commonly used adjective in the pattern. For instance, surprisingly, possible, which is quite commonly used in this pattern in the IS RAs, occurs in only 2 concordance lines in the GS RAs, both examples found in the Sociology RAs, one of which is (7).

7) Also males recognize symptoms of most STIs more easily than females; therefore it is possible that some girls may be harbouring infections without knowing that they are indeed infected. [SOC GH19]

My analysis did not reveal any corpus examples of this pattern in the Economics and Law RAs written by the GS.

Furthermore, the adjective form likely in this epistemic pattern is less productive in the Ghanaian RAs than it is in the international ones. While I found no corpus examples for Law, it occurs only once in Sociology and 3 times in Economics. Example (8) is one of the rare cases in the Ghanaian Economics RAs.

8) As stated earlier, it is likely that a significant number of Chinese investors come into Ghana with capital provided by the Chinese government and this would definitely give them an advantage. [ECO GH17]

Another noticeable feature in the international RAs which is absent in the Ghanaian RAs has to do with the negation of likely in this pattern, which, as I have noted, tend to reduce the epistemic strength from probability to one of doubt or impossibility. The concordance analysis does not reveal any examples in the Ghanaian sub-corpus of RAs of this negative pattern associated with likely, be it the clause negation type (not likely) or the derivational affix type (unlikely).

I have already established that the Ghanaian writers show a strong preference for the use of clear in the phraseological pattern being explored in this paper, especially in the fields of Law and Economics where the pattern is seen in several concordance lines. Indeed, there are 19 concordance lines for the it v-link clear that pattern in the Ghanaian Law RAs,
17 in Economics and 2 in Sociology. Thus overall, the frequencies suggest that the occurrence of clear in this pattern is even preferred by the Ghanaian writers more than their international colleagues. Figure 3 is the 17 concordance lines of clear in this pattern in the Ghanaian Economics RAs.

A close inspection of these lines (and those of the GS Law RAs) reveals some interesting qualitative peculiarities in the RAs produced by the Ghanaian writers when compared with the RAs written by the native-speaking IS. There is a tendency for the GS to (unconventionally) exert extreme force in the communication of research claims when deploying this pattern. I have already discussed how this tendency is manifested in a number of ways in their writing, including the underuse of possible in the it v-link ADJ that-clause pattern; the absence of negative likely in this pattern, which reduces the level of certainty; and the dominance of the strong epistemic form clear. Still yet, there is the unusual use of intensifiers such as very, starkly and abundantly to further enhance the epistemic strength of clear in this pattern, as in examples (9) and (10).

9) It is starkly clear that China has become increasingly involved in sub-Saharan Africa (SSA) over the past decade. [ECO GH17]

10) It is abundantly clear that English Courts scrutinize guarantees whenever there is a challenge before they can be enforced. [LAW GH20]

This kind of intensification of an-already strong epistemic device does not occur at all in the IS RAs, indicating that it is not a particularly acceptable and/or conventional way of deploying such intensifiers to boost research claims in mainstream discourse communities, perhaps because it overly or unnecessarily stresses authorial commitment. The evidence here seems to give support to research (e.g., Hyland \& Milton's 1997; Flowerdew, 1999; Hinkel, 2002, 2005) that argues that, for many an L2 writer, it is an enormous problem applying the appropriate degrees of certainty to make claims, as a common tendency is for them to overstate or exaggerate such claims.

\section{CONCLUSION}

In this article, I discuss the adjectives possible, likely and clear as epistemic devices in the phraseological pattern it 


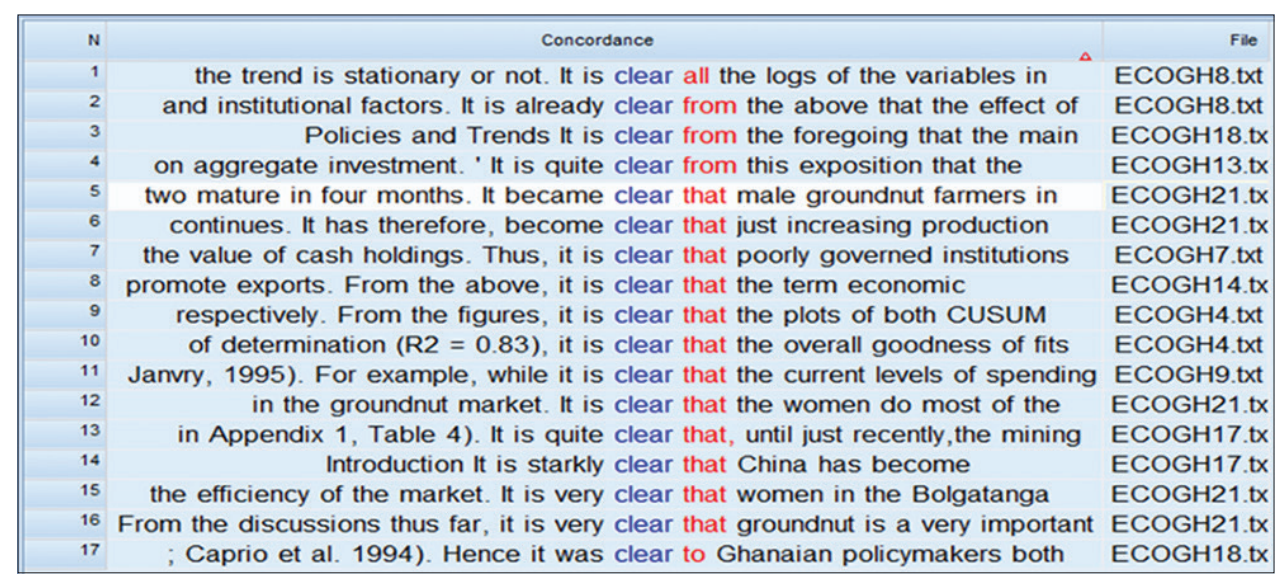

Figure 3. Concordance lines for the pattern it v-link clear that in the GS RAs

$v$-link ADJ that clause. Because the pattern is a very important rhetorical convention in mainstream scholarly writing, I rely on corpus methods to explore the ways that Ghanaian professional L2 writers in the fields of Sociology, Economics and Law use this phraseological pattern in their RAs, and to examine the extent to which their use of the pattern reflects international discourse community norms and practices.

The present study reveals that Ghanaian writers in all the three disciplines studied are aware of, and use, the adjectives possible, likely and clear in the pattern it v-link ADJ that in their RAs; at the same time, the study reveals a number of ways their use of this pattern diverges from international conventional practices, for which some adjustment might be necessary in order to adhere to the rhetorical requirements in mainstream international communities. First, there is an underuse of the pattern it v-link ADJ that (particularly with possibly and likely) and an overuse of the pattern with clear. This implies that these Ghanaian writers tend to hedge less and boost more argumentative claims in their RAs by focusing "more on the use of possible and likely in the co-occurrence pattern: it v-link ADJ that" (Ngula, 2015: 300). Second, the tendency for the L2 Ghanaian scholars to use already-strong epistemic devices (specifically 'clear' in this study) in the company of other intensifiers is not a feature of international rhetorical styles and needs to be discouraged. Expressions like starkly clear, abundantly clear and very clear found in the pattern it v-link ADJ that in the corpus of RAs written by the Ghanaian scholars "are not conventional in international disciplinary communities" (Ngula, 2015: 300).

A useful implication arising out of the findings reported here is that the dynamism that comes with the writing of rhetorically apt RAs in respected international Anglophone journals requires constant 'situated learning' (Lavor and Wenger, 1991). As Hyland (2001: 209) notes, such conscious learning, undertaken especially by less experienced users of English as an additional language who are seeking to publish in top English-medium journals, can help writers to conform to "the rhetorical conventions and social understandings of the community" and apply "suitable patterns of social and rhetorical interactions" when they write RAs. The Ghanaian writers studied in this article can benefit considerably from such on-the-job learning by averting their minds to the ste- reotypical rhetorical practices in these journals. This might require authorities in the institutions where these scholars work to ensure that such top-rated journals are readily accessible to faculty.

\section{REFERENCES}

Adika, G. S. K. (1999). An Analysis of University Students' Expository Discourse. Unpublished PhD thesis, University of Ghana.

Afful, J. B. A. (2005). The Rhetoric of Undergraduate Student Writing in a Ghanaian University: A Cross-disciplinary Study. Unpublished $\mathrm{PhD}$ thesis, National University of Singapore.

Ağçam, R. (2014). A corpus-based study on epistemic adjectives in academic English. American Journal of Educational Research, 2(12), 1230-1236.

Anyidoho, L. A. (2002). Analysis of the writing of final year students. Ghanaian Journal of English Studies, 1: $58-72$.

Biber, D., Johansson, S., Leech, G., Conrad, S. and Finegan, E. (1999). Longman Grammar of Spoken and Written English. London: Longman.

Biber, D., Conrad, S. \& Leech, G. (2002). Longman Student Grammar of Spoken and Written English. Harlow: Longman.

Canagarajah, S. (1996). Nondiscursive requirements in academic publishing, material resources of periphery scholars, and the politics of knowledge production. Written Communication, 13(4), 435-472.

Canagarajah, S. (2002). The Geopolitics of Academic Writing. Pittsburgh: University of Pittsburgh Press.

Charles, M. (2006). Phraseological patterns in reporting clauses used in citation: a corpus-based study of theses in two disciplines. English for Specific Purposes, 25, 310-331.

Coates, J. (1983). The Semantics of the Modal Auxiliaries. London: Croom Helm.

Dako, K. (1997). Features of stylistic versatility in English as observed in the writing of English graduates. In M. E. K. Dakubu, English in Ghana (pp. 55-68). Accra: Black Mask. 
Dunning, T. (1993). Accurate methods for statistics of surprise and coincidence. Computational Linguistics, 19(1), 61-74.

Englander, K. (2006). Revision of scientific manuscripts by nonnative-English-speaking scientists in response to journal editors' criticism of the language. Journal of Applied Linguistics, 3(2), 129-161.

Flowerdew, J. (1999). Problems in writing for scholarly publication in English: the case of Hong Kong. Journal of Second Language Writing, 8(3), 243-264.

Groom, N. (2005). Pattern and meaning across genres and disciplines: an exploratory study. Journal of English for Academic Purposes, 4, 257-277.

Hinkel, E. (2002). Second Language Writers' Text: Linguistic and Rhetorical Features. Mahwah: Lawrence Erlbaum Associates.

Hinkel, E. (2005). Hedging, inflating and persuading in L2 academic writing. Applied Language Learning, 24(2), 29-54.

Hunston, S. \& Francis, G. (2000). Pattern Grammar: A Corpus-Driven Approach to the Lexical Grammar of English. Amsterdam: John Benjamins.

Hunston, S. (2002). Corpora in Applied Linguistics. Cambridge: Cambridge University Press.

Hunston, S. (2011). Corpus Approaches to Evaluation: Phraseology and Evaluative Language. New York: Routledge.

Hyland, K. \& Milton, J. (1997). Qualification and certainty in L1 and L2 students' writing. Journal of Second Language Writing, 6(2), 185-205.

Hyland K. (2001). Definitely a possible explanation: epistemic modality in academic argument. In M. Gotti \& M. Dossena, Modality in Specialized Texts (pp. 291-230). Bern: Peter Lang.

Laso, N. J. \& John, S. (2013). A corpus-based analysis of the collocational patterning of adjectives with abstract nouns in medical English. In I. Verdaguer, N. J. Laso \& D. Salazar, Biomedical English: A Corpus-Based Approach (pp. 55-71), Amsterdam: John Benjamins.

Laso, N. J., Comelles, E. \& Verdaguer, I. (2013). Negation in biomedical English. In I. Verdaguer, N. J. Laso \& D. Salazar, Biomedical English: A Corpus-Based Approach (pp. 105-119). Amsterdam: John Benjamins.

Lave, J. \& Wenger, E. (1991). Situated Learning: Legitimate Peripheral Participation. Cambridge: Cambridge University Press.

Leki, I., Cumming, A. \& Silva, T. (2008). A Synthesis of Research on Second Language Writing in English. New York: Routledge.

Martinéz, I. A. (2005). Native and non-native writers' use of first person pronouns in the different sections of biology research articles in English. Journal of Second Language Writing, 14, 174-190.
McEnery, T. \& Kifle, N. A. (2002). Epistemic modality in argumentative essays of second-language writers. In J. Flowerdew, Academic Discourse (pp. 182-195). London: Longman.

McEnery, T., Xiao, R. \& Tono, Y. (2006). Corpus-Based Language Studies: An Advanced Resource Book. London: Routledge.

Ngula, R. S. (2015). Epistemic Modality in Social Science Research Articles Written by Ghanaian Authors: A Corpus-Based Study of Disciplinary and Native vs. Non-Native Variations. Unpublished $\mathrm{PhD}$ thesis, Lancaster University.

Nkemleke, D. (2010). Cameroonian and foreign scholars' discourse: the rhetoric of conference abstracts. World Englishes, 29(2), 173-191.

Nuyts, J. (2001). Epistemic Modality, Language and Conceptualization: A Cognitive-Pragmatic Perspective. Amsterdam: John Benjamins.

Panocová, R. (2008). Expressions of modality in biomedical texts. SKASE Journal of Translation and Interpretation, 3(1), 82-90.

Peacock, M. (2012). High-frequency collocations of nouns in research articles across eight disciplines. Ibérica, 23, $29-46$.

Römer, U. (2009). The inseparability of lexis and grammar: corpus linguistic perspectives. Annual Review of Cognitive Linguistics, 7, 141-163.

Salager-Meyer, F. (2008). Scientific publishing in developing countries: challenges for the future. Journal of English for Academic Purposes, 7, 121-132.

Sinclair, J. (1987). Collocation: a progress report. In R. Steele \& T. Threadgold, Language Topics: An International Collection of Papers by Colleagues, Students and Admirers of Professor Michael Halliday to Honour him on his Retirement (pp. 319-331), Vol. II Amsterdam: John Benjamins.

Sinclair, J. (1991). Corpus, Concordance and Collocation. Oxford: Oxford University Press.

Sinclair, J. (2004). Trust the Text: Language, Corpus and Discourse. London: Routledge.

Sinclair, J. (2008). Preface. In S. Granger \& F. Meunier, Phraseology: An Interdisciplinary Perspective (pp. xvixviii), Amsterdam: John Benjamins.

Swales, J. M. (2004). Research Genres. Cambridge: Cambridge University Press.

Ventura, A. (2013). As described below: a corpus-based approach to the verb describe in scientific English. In I. Verdaguer, N. J. Laso \& D. Salazar, Biomedical English: A Corpus-Based Approach (pp. 73-104). Amsterdam: John Benjamins.

Vold, E. T. (2006). The choice and use of epistemic markers in linguistics and medical research articles. In K. Hyland \& M. Bondi, Academic Discourse Across Disciplines (pp. 225-249). Bern: Peter Lang. 